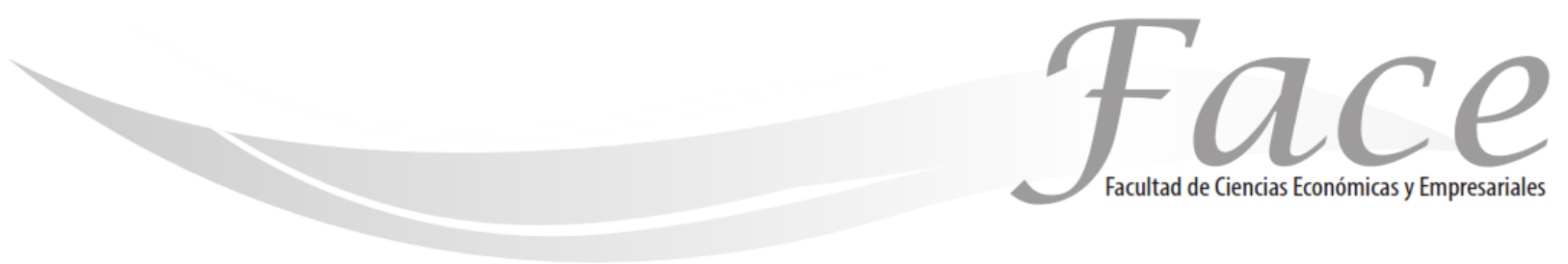

ISSN Impreso: 1794-9920 ISSN Electrónico: 2500-9338

Volumen 18-N¹

Año 2018

Págs. $75-81$

\title{
LA CARTOGRAFÍA SOCIAL Y EL USO DE LOS SISTEMAS DE INFORMACIÓN GEOGRÁFICA S.I.G. COMO ESTRATEGIA PARA LA ENSEÑANZA DE LA GEOGRAFÍA EN LA UPEL- IPRGR.
}

\author{
Fernando José Ramírez Morales * \\ Enlace ORCID: https://orcid.org/0000-0001-9934-4169 \\ Luis Daniel Gualdrón Guerrero ** \\ Enlace ORCID: https://orcid.org/0000-0001-5024-1185
}

Fecha de Recepción: 27 de Diciembre 2017

Fecha de Aprobación: 5 de Abril 2018

\section{Resumen:}

El presente estudio está dirigido a analizar las estrategias empleadas a la hora de enseñar geografía, con el propósito de proponer el uso de la cartografía social y los sistemas de información geográfica S.I.G. para la enseñanza de la realidad local en la formación de docentes del área de geografía en la Universidad Pedagógica Experimental Libertador, instituto pedagógico rural Gervacio Rubio (UPEL-IPRGR).

Palabras Clave: Estrategias, educación, enseñanza de la geografía, cartografía social, sistemas de información geográfico.

\footnotetext{
* Profesor especialista en geografía e Historia, Magister en Educación mención enseñanza de la Geografía, Doctorando en Ciencias de la Educación. Profesor de Sociología del Instituto Pedagógico Rural Gervasio Rubio, UPEL. Coordinador del Centro de Información Geográfica UPEL IPRGR, Coordinador de la line de investigación, La Enseñanza de la Geografía en los Tiempos Actuales. Correo Electrónico: luisdanielgualdronguerrero@gmail.com

**. MSc. en Planificación Global, Doctorando en ingeniería ambiental en la Universidad del Zulia, Venezuela, en la línea de investigación en Desarrollo y Optimización de Técnicas Analíticas en las Ciencias Ambientales. Correo Electrónico: luisdanielgualdronguerrero@gmail.com
} 
THE SOCIAL CARTOGRAPHY AND THE USE OF GEOGRAPHICAL INFORMATION SYSTEMS S.I.G. AS A STRATEGY FOR THE TEACHING OF GEOGRAPHY IN UPEL- IPRGR.

\begin{abstract}
:
The present study is aimed at analyzing the strategies used when teaching geography, with the purpose of proposing the use of social cartography and geographic information systems S.I.G. for the teaching of local reality in the training of doctoral students in the area of geography at the Pedagogical Experimental University Liberator, rural pedagogical institute Gervacio Rubio (UPELIPRGR).
\end{abstract}

Keywords: Strategies, education, geography teaching, social cartography, geographic information systems.

\title{
CARTOGRAFIA SOCIAL E USO DE SISTEMAS DE INFORMAÇÃO GEOGRÁFICA S.I.G. COMO ESTRATÉGIA PARA O ENSINO DA GEOGRAFIA EM UPPAL- PRGR.
}

Resumo:

O presente estudo tem como objetivo analisar as estratégias utilizadas no ensino de geografia, com o objetivo de propor o uso de sistemas de cartografia social e de informação geográfica S.I.G. para o ensino da realidade local na formação de professores da área de geografia na Universidade Pedagógica Experimental Libertador, do instituto pedagógico rural Gervacio Rubio (UPELIPRGR).

Palavras chave: Estratégias, educação, ensino de geografia, cartografia social, sistemas de informação geográfica. 
Fernando José Ramírez Morales - Luis Daniel Gualdrón Guerrero

\section{INTRODUCCIÓN:}

El sistema Educativo venezolano y, particularmente, el nivel de educación superior, se observa que el mismo ha estado disociado de la realidad, producto de su aplicación de prácticas pedagógicas controladas, centralizadas y sujetadas a lineamientos externos de diversa índole. Por consiguiente, es importante aplicar en la educación superior, estrategias que promuevan nuevas alternativas de cambio, este trabajo se orienta al estudio de las ventajas que ofrece el uso de la cartografía social y los sistemas de información geográfica S.I.G. para la enseñanza de la geografía, en correspondencia con el contexto local, a fines de producir formas de enseñanza alternativas que hagan de las clases una acción sustentada en el abordaje de los contenidos de manera integrada.

\section{MARCO TEÓRICO :}

\section{La cartografía social y el uso de los S.I.G. como estrategias en la enseñanza de la geografía.}

Las complejas situaciones que enfrenta la sociedad en la actualidad, hacen que sea necesario la aplicación de nuevas técnicas y estrategias de análisis que puedan dar interpretación alguna a las corrientes que configuran y controlan el mundo, es en este plano donde surge la cartografía social como una nueva herramienta de comprensión de las relaciones que se gestan entre las sociedades y los humanos, bajo las disposiciones planteadas anteriormente. Por ello, resulta interesante la inclusión de estas teorías modernas que buscan explicar desde perspectivas más centradas en el contexto local las circunstancias relevantes para el que hacer geográfico y cartográfico. Fernández y otros (2011) definen la cartografía social de la siguiente forma:

La cartografía social es, entonces un camino para el reconocimiento del mundo cultural, ecológico, productivo y político que se expresa en el territorio y que nos debe servir como ubicación dentro de un contexto social que nos permita construir un presente y el futuro (p. 03)

Es evidente, que las preocupaciones de la cartografía social giran en torno a lo que la sociedad misma es capaz de producir basados en sus identidades culturales las cuales son descritas por Mosquera y Reyes (2013). como el conjunto de valores, orgullo, tradiciones, símbolos, creencias y modos de comportamiento que funcionan como elementos dentro de un grupo social y que actúan para que los individuos que lo forman puedan fundamentar su sentimiento de pertenencia, que hacen parte de la diversidad al interior de las mismas en respuesta a los intereses, rituales que comparten dichos grupos dentro de la cultura dominante (p.156)

Usándola como recurso fundamental de planificación y trabajo de los espacios locales. Entendiendo que la planificación espacial, es vital para el desarrollo de las comunidades y de allí se deriva la importancia de implementar este nuevo enfoque, sin embargo, la cartografía social también centra su atención en el producto final de su intervención, sobre el cual realiza un proceso de reflexión entre la conexión y el intercambio de vivencias que marcaron el mismo.

Desde esta perspectiva, la cartografía social va a ser una herramienta de uso colectivo para interpretar la realidad socio-económico e histórico-cultural y sin duda alguna la espacial. Aunado a esto, se ha intentado incluir la tecnología dentro de esta nueva tendencia cartográfica, donde el S.I.G. implementándolo en la sociedad y en la escuela pueden en primer lugar promover la espacialidad y la reconstrucción del territorio, y en segundo lugar tecnológicamente motivar al desarrollo de clases dinámicas e interactivas, logrando generar nuevos métodos de enseñanza y aprendizaje. Por otra parte, surgen interrogantes acerca de los sistemas de información geográfica (S.I.G.), ante ello, Bernal y Galindo (2012) plantean que:

El término S.I.G. se establece de la palabra en inglés Geographic Information System (GIS). Se le define como una herramienta de software que nos permite almacenar, recuperar, analizar y desplegar información geográfica. Éste tiene la capacidad de efectuar una gestión completa de datos referenciados geográficamente. Por datos similares que indica a los datos geográficos o mapas que constan de coordenadas geográficas reales asociadas (p.169).

Actualmente, una de las formas más sencillas de acceder a la localidad lo representa el uso de la tecnología, y es allí donde los sistemas de información geográfica (S.I.G.) deben dar respuestas oportunas de la realidad inmediata, estas a su vez giran en torno a datos mayormente 
ISSN: 1794-9920 - ISSN Electrónico: 2500-9338

Enero - Julio de 2018

Volumen 18 Número 1, Año 2018 Págs. 75 - 81

estadísticos, producto de la sistematización y digitalización del territorio.

Lo que conlleva a que el proceso de análisis de la información contenida en los S.I.G. sea un proceso separado y es allí donde la cartografía social encuentra cabida dentro de esta dualidad.

Entonces resulta como punto esencial de la enseñanza de la geografía resaltar el valor de las nuevas tendencias teóricas que intentan abordar lo complejo de las relaciones hombre-medio.

Esta visión renovada, es la que debe ser plasmada en el ámbito educativo a la hora de enseñar la ciencia geográfica, ya que intenta hacer una deconstrucción de la realidad social, el complejo y basto mundo que las componen, así como también intentar reflexionar sobre las relaciones generadas con el espacio geográfico. Es de interés, para la educación incluir en primer lugar la tecnología S.I.G. y en segundo lugar los análisis oportunos de la cartografía social.

\section{METODOLOGÍA:}

La presente investigación se enfocó en el paradigma cualitativo, motivados por el interés de revisar las cualidades presentes en el acto educativo a la hora de enseñar. Donde se busca tratar de entender y explicar una realidad social existente y analizar las posibles consecuencias que la misma genera, ahora bien, es necesario centrar la atención en la razón de ser del paradigma ya que el mismo se ajusta a los criterios de la presente investigación, cuya finalidad no es más que a la luz de la razón dar alternativas educativas a la hora de enseñar Geografía, ante ello, Denzin y Lincoln (1994) plantean lo siguiente:

La investigación cualitativa es una actividad sistemática orientada a la comprensión en profundidad de fenómenos educativos y sociales, a la transformación de prácticas y escenarios socioeducativos, a la toma de decisiones y también hacia el descubrimiento y desarrollo de un cuerpo organizado de conocimientos (p. 123)

En función a lo expuesto, es resaltante que en principio la investigación cualitativa busca de forma subjetiva la elaboración conceptual y el desarrollo teórico en función a las vivencias directas, sentidas y experimentadas al momento de realizar la investigación, entendiendo que dichas vivencias son las encargadas del proceso de transformación del acto educativo específicamente, y esto se debe a que se busca integrar los hallazgos de la realidad con las percepciones teóricas planteadas, con el fin de dar explicación a las realidades imperantes en la sociedad.

Pues como lo indica Bisquerra (1989): "Es una investigación desde dentro, que supone una preponderancia de lo individual y subjetivo. Su concepción de la realidad social entra en la perspectiva humanística" (p.23). Lo que afianza la idea de que es una investigación interpretativa, referida inicialmente al individuo, a lo particular y es a partir de ello que se llega a realizar una posible generalización, contrastado a teorías. En consecuencia, la didáctica empleada por el docente se estudia en la presente investigación a partir de particularidades. Por tanto, prevalecerá la interpretación a la luz de la constatación con los fundamentos teóricos del estudio y las acciones cotidianas que realizan los individuos en la construcción social del proceso de enseñanza y aprendizaje específicamente en el que hacer geográfico.

En función a lo planteado, lo que se busca con esta investigación es comprender la delineación, el análisis e interpretación de los aspectos más relevantes contenidos en las categorías que son sometidas a objeto de estudio, es decir, a la cotidianidad misma contenida en los ambientes de clases donde se geste el proceso de enseñanza. Para de estar forma centrar la atención sobre la solución teórica y práctica a fin de intentar mejorar 0 cambiar drásticamente las situaciones vividas por los grupos de individuos que se involucran en la misma; buscando predecir los resultados que arroje las situaciones sometidas en cuestión. A su vez, esta investigación corresponde a la modalidad de proyecto factible, definido por el manual de trabajos de grado de la UPEL (2006) como:

\footnotetext{
Un estudio que consiste en la investigación, elaboración y desarrollo de una propuesta de un modelo operativo viable para solucionar problemas, requerimientos o necesidades de organizaciones 0 grupos sociales... la propuesta que lo define puede referirá la formulación de políticas, programas, tecnologías, métodos o procesos, que solo tienen sentido en el ámbito de sus necesidades (p.07)
}

Lo que permite aludir, que un proyecto en la modalidad de factible pretende 0 se orienta en la resolución de problemas buscando la satisfacción de necesidades en un contexto determinado, cuyas pretensiones giran en torno a realizar una propuesta ajustada con la realidad encontrada, centrándose en un tipo de investigación orientada en decisiones, al respecto, Tamayo (1999) la define como 
Fernando José Ramírez Morales - Luis Daniel Gualdrón Guerrero

"aquella investigación cuyo fin inmediato es la creación de alternativas de solución, que de forma directa ya que se podrá realizar un estudio de tal situación en el lugar de los hechos" (p. 17), el conocimiento de la realidad educativa y geográfica, permitirá presenciar los acontecimientos suscitados en ese espacio vivido, y al contrastar la realidad local se podrá tener un manejo de la información mucho más acertada.

\section{RESULTADOS Y DISCUSIÓN:}

Categoría emergente: Enseñanza de la Geografía desde perspectivas tradicionales

\section{Sub categoría emergente: Uso del mapa.}

El uso del mapa se encuentra por así decirlo, limitado exclusivamente a la ubicación de lugares, lo que indica que desde perspectivas teóricas se enseña a producir mapas para luego usarlos únicamente para ubicar un contexto determinado. En cuanto a las tendencias emergentes, el uso del mapa debe ser comprendido como el medio dinamizante de las clases de geografía, es decir, debe estar cargado de alto contenido pedagógico y que el mismo, involucre a los estudiantes en el análisis de todas las situaciones contenidas en él.

Es decir, el uso del mapa se ha resumido a una especie de inventario de todos los elementos incluidos en el; el desfase que existe entre la realidad educativa y lo planteado desde la actualidad invita a pensar y repensar el hecho de la viabilidad que tiene el mapa como vía de enseñanza.

\section{Categoría emergente: Los sistemas de información geográfica en la enseñanza de la geografía}

Sub categoría emergente: Los sistemas de información geográfica como estrategia de enseñanza.

\author{
Según Nieto (2010): \\ La utilización de los Sistemas de Información \\ Geográfica aporta la doble vertiente de adquisición \\ de destrezas y fomento de valores educativos, \\ culturales, ambientales, al trabajar con fuentes de \\ información de distinta índole. (p. 158)
}

La enseñanza de la geografía, se ha visto influida por la implementación de nuevas posturas tecnológicas que promueven el desarrollo del saber científico desde perspectivas didácticas; el uso de los S.I.G. como medio para propiciar el aprendizaje, permite ampliar la visión crítica que se pretende implementar como elemento que propicie el despertar pedagógico del que hacer geográfico y que esto a su vez de paso a la consolidación de dicha disciplina científica bajo el rigor que los momentos actuales lo demandan, aunado a ello es pertinente acotar que para el proceso de diseño y elaboración de mapa los S.I.G. representan la alternativa más viables para satisfacer las necesidades que dicho proceso se plantea.

\section{Sub categoría emergente: Uso de los sistemas de información geográfica en la enseñanza de la geografía.}

Uno de los elementos de mayor relevancia dentro de las clases de cartografía radica en el gran cuestionamiento que gira en torno al uso de los S.I.G. como estrategia de enseñanza. Sarria (2009) plantea que: "las funciones más usada de un SIG son almacenamiento, visualización, y análisis de datos espaciales. Un uso más avanzado sería la utilización de un SIG para la toma de decisiones en ordenación territorial 0 para supervisión de procesos ambientales" (p. 41).

Categoría emergente: La cartografía social y el uso de los S.I.G. como estrategia para enseñar geografía

Sub categoría emergente: Cartografía social y uso de los S.I.G. nuevas Perspectivas de enseñanza.

Montes (2008) plantea que "los SIG, permiten acceder a todos los entornos, y partiendo de estas experiencias se posibilita realizar una lectura de los diferentes elementos que configuran un espacio geográfico, de las interrelaciones que entre ellos se dan y de su distribución" (p. 55). En tal sentido, la cartografía social ligada al uso de los S.I.G, surge como una tendencia que revaloriza el papel de la geografía en el plano social y que busca involucrar a todos los miembros de la misma en dicha construcción.

\section{PROGRAMA DE PROFUNDIZACIÓN, AMPLIACIÓN Y CARTIFICACIÓN EN CARTOGRAFÍA SOCIAL Y SISTEMAS DE INFORMACIÓN GEOGRÁFICA PARA EL PERSONAL ACADÉMICO DE EDUCACIÓN SUPERIOR}

\section{Justificación Pedagógica}

la presente propuesta se fundamenta en el área de las Ciencias Sociales, específicamente tiene en cuenta contenidos relacionados con la Geografía, debido a que resulta perentorio enseñar en este nivel académico dichos contenidos a partir del uso de la Cartografía Social y los S.I.G. para fomentar en los estudiantes el interés y motivación en reconocer los rasgos de la realidad geográfica local, para comprender la dinámica mundial que se experimenta en la actualidad. 
Por lo tanto, se plantean una serie de cursos y/o talleres, que buscan promover las nuevas tendencias del saber geográfico. Permitiendo que a partir de ellos los docentes sean capaces de generar diversas estrategias innovadoras fundamentadas en la cartografía social y el uso de los S.I.G. dirigidas específicamente a los momentos de una clase.

\section{Estructura de la Propuesta}

La presente propuesta estará dirigida a los docentes que laboran en la UPEL-IPRGR en el área de geografía. Está estructurada en una fase totalmente formativa: ella contiene el objetivo general o propósito de la propuesta, desarrollado, desde la aplicación de cursos no conducentes a título que mejoren el quehacer y la experiencia docente y que permita el fortalecimiento del uso de la cartografía social y de los S.I.G

\section{Fase de la Propuesta. Formativa}

\section{Objetivo General o Propósito}

Fortalecer el uso la cartografía social y de los sistemas de información geográfica para el estudio de la realidad local en el programa de Postgrado de la UPEL-IPRGR.

\section{Presentación}

La cartografía social busca en lo posible la intervención de otras esferas sociales más allá de lo local. Ante lo planteado, va a ser la universidad en su carácter social la encargada de contribuir, en la inclusión de nuevas tendencias geográficas que promuevan el desarrollo de las sociedades, en función a la interpretación que se les da a los fenómenos contenidos en las mismas, explotando el rol de investigador de los docentes que estudian y laboran en su campo de acción. En síntesis, se presenta un programa dirigido a fortalecer las competencias del docente a la hora de enseñar geografía.

\section{Descripción}

El programa consta de dos (2) módulos: la realidad local, en síntesis, cartografía social, diagnostico comunitario, elaboración de mapas o cartogramas (módulo l) y levantamiento de información territorial usando "GPS", procesamiento de dicha información con S.I.G., elaboración de cartografía temática y geohistórica digital (módulo II) 
Fernando José Ramírez Morales - Luis Daniel Gualdrón Guerrero

La implementación de la cartografía social y los S.I.G. como se mencionó a lo largo de la investigación, representaría una garantía de la consolidación del proceso de enseñanza, transformando la forma como se enseña el saber geográfico, reestructurando la forma de ver y comprender la realidad, es por ello, que desde estas aproximaciones es factible aplicar, la cartografía social y el uso de los S.I.G. como estrategia para la enseñanza de la geografía en la UPEL-IPRGR.

\section{REFERENCIAS:}

Bernal, R. Galindo, D. (2012). Cartografía social y sistemas de información geográfica, una nueva experiencia en la educación. Geosig, año 4.

Bisquerra, R. (1989). Métodos de investigación educativa. Barcelona. España. Ediciones CEAC.

Universidad Pedagógica Experimental Libertador (2006) Manual de trabajos de grado de especialización y maestría y tesis doctorales. Segunda reimpresión. Caracas Venezuela, FONDE editorial UPEL.

Denzin Y Lincoln (1994). Handbook of Qualitative Research. (2nd Edition) Londres: Sage Publications.

Fernández y otros (2011) SIG-P y Experiencias de Cartografía Social en la ciudad de Bogota (COLOMBIA). Universidad Nacional de Colombia.

Montes, C. (2008). Los Sistemas de Información Geográfica como medio didáctico en la enseñanza de la geografía. Universidad de Antioquia, Medellín, Colombia.

Mosquera Jemay y Reyes celesky (2013), La valoración y recuperación de saberes como instrumento para la construcción de territorio y el desarrollo endógeno, FACE, volumen $13,151-170$ páginas.

Nieto, A. (2010). El uso didáctico de los sistemas de información geográfica en el Espacio Europeo de Educación Superior. Universidad de Extremadura.

Sarria, F. (2009). Sistemas de Información Geográfica. Madrid, España

Tamayo (1999). El Proceso de la Investigación. Limusa Noriega Editores, Tercera Edición. 